PROCEEDINGS OF THE

AMERICAN MATHEMATICAL SOCIETY

Volume 131, Number 4, Pages 1205-1214

S 0002-9939(02)06679-0

Article electronically published on July 26, 2002

\title{
EIGENVALUE ASYMPTOTICS OF PERTURBED PERIODIC DIRAC SYSTEMS IN THE SLOW-DECAY LIMIT
}

\author{
KARL MICHAEL SCHMIDT
}

(Communicated by Joseph A. Ball)

\begin{abstract}
A perturbation decaying to 0 at $\infty$ and not too irregular at 0 introduces at most a discrete set of eigenvalues into the spectral gaps of a one-dimensional Dirac operator on the half-line. We show that the number of these eigenvalues in a compact subset of a gap in the essential spectrum is given by a quasi-semiclassical asymptotic formula in the slow-decay limit, which for power-decaying perturbations is equivalent to the large-coupling limit. This asymptotic behaviour elucidates the origin of the dense point spectrum observed in spherically symmetric, radially periodic three-dimensional Dirac operators.
\end{abstract}

\section{INTRODUCTION}

For a large class of potentials, the semiclassical Weyl formula gives a correct asymptotic description of the total multiplicity of the lower spectrum of a Schrödinger operator in the large coupling limit (see [18, Chapter XIII.15, and the references given there). However, there are also some notable exceptions, e.g. in the twodimensional case $(9],[10])$. In recent years an analogous asymptotic analysis of the point spectrum arising in spectral gaps of Schrödinger operators under perturbations has attracted considerable attention; starting from [1, Birman has developed a general framework to study this problem [2], [3], 4], [5], 6], [7]; see also [16].

More specifically, Sobolev [26] has studied the perturbed periodic one-dimensional Schrödinger operator, showing that for a wide range of power-decaying perturbations, the number of eigenvalues arising in a closed subinterval of a spectral gap of the unperturbed problem is asymptotically given by a quasi-semiclassical formula in which the quasimomentum of the periodic background problem takes the role of the ordinary momentum in the Weyl formula. This result has an interesting application to spherically symmetric Schrödinger operators in $\mathbb{R}^{n}$ with a radially periodic potential, which have dense point spectrum in all spectral gaps of the corresponding one-dimensional periodic operator [14. Indeed, treating the angular momentum term of the one-dimensional half-line operators in the partial-wave

Received by the editors August 3, 2001 and, in revised form, November 16, 2001.

2000 Mathematics Subject Classification. Primary 34L20, 34L40, 47E05, 81Q10, 81Q15.

(C)2002 American Mathematical Society 
decomposition

$$
\begin{aligned}
-\Delta+q(|\cdot|)= & \bigoplus_{l \in \mathbb{N}_{0}}-\frac{d^{2}}{d r^{2}}+q(r) \\
& +\left(l(l+n-2)+\frac{(n-1)(n-3)}{4}\right) / r^{2} \quad(r \in(0, \infty)),
\end{aligned}
$$

with periodic $q$, as a perturbation, the quasi-semiclassical formula explains the origin of the dense eigenvalues, and describes their asymptotic density in the limit $l \rightarrow \infty$. Furthermore, numerical experiments have shown that the asymptotic formula can give a surprisingly accurate prediction for the number of eigenvalues, even for small values of the coupling constant [11. (We mention in passing that the angular momentum term also happens to be a boundary case for the question of whether the total number of eigenvalues introduced in a spectral gap for an individual half-line operator is finite or infinite ([19], [20], 21], 22], 25]); but this critical behaviour is not apparent in the large-coupling limit.)

The relativistic counterpart of the Schrödinger operator, the Dirac operator, is unbounded below, so that there is no lower spectrum, and one is always in a gap situation when studying the discrete spectrum, even when there is no periodic background potential (for the large coupling asymptotics in that case see e.g. [8], which corrects a result by [15, and [12]). Again, an interesting class of perturbed periodic one-dimensional operators arises from the partial-wave decomposition of the spherically symmetric, radially periodic Dirac operator in $\mathbb{R}^{3}$,

$$
H=-i \alpha \cdot \nabla+m \beta+q(|\cdot|) \cong \bigoplus_{k \in \mathbb{Z} \backslash\{0\}}-i \sigma_{2} \frac{d}{d r}+m \sigma_{3}+q(r)+\frac{k}{r} \sigma_{1}
$$

where $\alpha_{1}, \alpha_{2}, \alpha_{3}, \beta$ are $4 \times 4$ Dirac matrices, and

$$
\sigma_{1}=\left(\begin{array}{cc}
0 & 1 \\
1 & 0,
\end{array}\right) \quad \sigma_{2}=\left(\begin{array}{cc}
0 & -i \\
i & 0,
\end{array}\right) \quad \sigma_{3}=\left(\begin{array}{cc}
1 & 0 \\
0 & -1
\end{array}\right) .
$$

This operator also has dense point spectrum in the spectral gaps of the corresponding one-dimensional periodic operator, but one exceptional gap may or may not contain dense point spectrum - it is an open question of whether partial filling of the gap can occur [23], 24]. The underlying reason for the complications in the Dirac case is the fact that the angular momentum term $\sigma_{1} k / r$, in contrast to the Schrödinger angular momentum term, does not have a definite sign. Since it does not depend monotonically on the quantum number $k$, the eigenvalues can move either way as $k$ increases. Methods such as Sturm comparison, which yields a fairly quick proof for the quasi-semiclassical formula for perturbed Sturm-Liouville operators, are of little use when applied to matrix perturbations of Dirac operators.

In the present paper we study the asymptotic distribution of eigenvalues in spectral gaps of periodic Dirac operators with a matrix perturbation of the type of the angular momentum term. We assume that the perturbation tends to 0 at $\infty$, but no assumptions on its sign or rate of decay are needed. The asymptotic limit we consider is in fact a 'slow-decay' limit, which for perturbations of inverse power decay is equivalent to the 'large-coupling' limit, an observation central to Sobolev's result. More explicitly, given a Dirac system

$$
\tau=-i \sigma_{2} \frac{d}{d x}+m \sigma_{3}+q(x)
$$


with $m>0$ and real-valued, $\alpha$-periodic $q \in L_{\text {loc }}^{1}(\mathbb{R})$, and a real-valued perturbation template

$$
l_{0} \in C(0, \infty), \quad \lim _{\varrho \rightarrow 0} l_{0}(\varrho)=\infty, \quad \lim _{\varrho \rightarrow \infty} l_{0}(\varrho)=0,
$$

we investigate the number of eigenvalues of the perturbed Dirac system on $(0, \infty)$

$$
\tau(c)=-i \sigma_{2} \frac{d}{d r}+m \sigma_{3}+q(r)+l_{0}(r / c) \sigma_{1}
$$

in a compact subinterval of a spectral gap asymptotically as $c \rightarrow \infty$.

The angular momentum term is clearly of this type, with $l_{0}(\varrho)=1 / \varrho$.

We follow Sobolev's basic idea of comparing $\tau(c)$ to an operator in which $l_{0}(r / c)$ is locally replaced by a constant, but in the absence of Sturm comparison to obtain upper and lower bounds we must resort to the coarser instrument of operator perturbation theory. This complication leads to severe difficulties near 0 , where the perturbation is divergent: in the Sturm-Liouville case this actually helps, as the perturbed eigenvalue equation becomes disconjugate near 0 , so that no spectrum is produced near that end-point. The case of the Dirac system, however, with an unperturbed operator unbounded below and a strong perturbation of no definite sign, is entirely different. For this reason we introduce the following additional assumptions on $q$ and $l_{0}$ to ensure that essentially no spectrum is created near 0 ; as will be seen, they also imply that $\tau(c)$ is in the limit point case at 0 for sufficiently large $c$.

$$
q \in L^{\infty}(\mathbb{R}) ; \quad l_{0} \in A C_{\mathrm{loc}}(0, \cdot), \quad \limsup _{\varrho \rightarrow 0} \frac{\left|l_{0}^{\prime}(\varrho)\right|}{l_{0}^{2}(\varrho)}<\infty .
$$

The requirements on $l_{0}$ are clearly satisfied in the case of the angular momentum term.

Let $k(\lambda, l)$ be the quasi-momentum (cf. Section 2 below) of the periodic equation

$$
\tau u=\left(\lambda-l \sigma_{1}\right) u,
$$

for $\lambda, l \in \mathbb{R}$.

Our main result is

Theorem 1. Assume that $(\mathrm{H})$ holds in addition to the general hypotheses, and let $\left[\lambda_{1}, \lambda_{2}\right]$ be compactly contained in a spectral gap of the self-adjoint realisation $h$ of $\tau$.

Then for sufficiently large $c>0, \tau(c)$ is essentially self-adjoint on $C_{0}^{\infty}(0, \infty)$, and the number $N(c)$ of eigenvalues in $\left[\lambda_{1}, \lambda_{2}\right]$ of its self-adjoint extension $h(c)$ satisfies

$$
\lim _{c \rightarrow \infty} \frac{N(c)}{c}=\frac{1}{\alpha \pi} \int_{0}^{\infty}\left(k\left(\lambda_{2}, l_{0}(\varrho)\right)-k\left(\lambda_{1}, l_{0}(\varrho)\right)\right) d \varrho .
$$

Remarks. 1. Our assumptions imply that the essential spectra of $h(c)$ and $h$ coincide, so $h(c)$ has only discrete eigenvalues in $\left[\lambda_{1}, \lambda_{2}\right]$. It will be apparent from the proof that the asymptotic formula in Theorem 1 continues to hold if, instead of $(\mathrm{H})$ and $\lim _{\varrho \rightarrow 0} l_{0}(\varrho)=\infty$, we assume $l_{0} \in C([0, \infty))$.

2. In [23], Theorem 2, it was shown that $\lambda \in \mathbb{R}$ is a point of the essential spectrum of $H$ if $\lambda$ is in the spectrum of the periodic operator $h+l \sigma_{1}$ for some $l \in \mathbb{R}$, i.e. if $\lambda$ is a point of growth of $k(\cdot, l)$. In a rather weak sense, Theorem 1 is a reverse of this statement: if a $\lambda$ interval does not intersect the spectrum of $h+l \sigma_{1}$ 
for any $l \in \mathbb{R}$, then the integral on the r.h.s. of the asymptotic formula vanishes, i.e. the asymptotic eigenvalue density is 0 . Of course, this does not rule out the existence of dense point spectrum of $H$ in this interval.

\section{Rotation NUMBER AND QUASIMOMENTUM OF PERIODIC DIRAC SYSTEMS}

The qualitative behaviour of solutions of a linear ordinary differential equation with periodic coefficients is characterised by its monodromy matrix, the value of the canonical fundamental system after one period. In the case of an $\alpha$-periodic SturmLiouville or Dirac equation, this is a $2 \times 2$ matrix with real entries and (Wronskian) determinant 1 ; the position of its eigenvalues $\mu_{1}, \mu_{2}$ in the complex plane is thus fully determined by its trace, the discriminant $D=\mu_{1}+\mu_{2}$. If $\mu_{1} \neq \mu_{2}$, then there are corresponding Floquet solutions $u_{1}, u_{2}$ satisfying

$$
u_{j}(x+\alpha)=\mu_{j} u_{j}(x) \quad(x \in \mathbb{R}, j \in\{1,2\}) .
$$

Hence it is easy to see that the equation is stable (all solutions are globally bounded) if $|D|<2$, and unstable (all solutions are unbounded) if $|D|>2$; as $D$ is an analytic function of the spectral parameter, the real line splits into alternating stability and instability intervals. This already provides a complete description of the (purely absolutely continuous) spectrum of the corresponding self-adjoint ordinary differential operator: it is the closure of the union of all stability intervals, spectral gaps corresponding to non-degenerate instability intervals (cf. [13, 27]).

Furthermore, the oscillation behaviour of solutions is very closely linked to the discriminant as well. Thus for the periodic Dirac system, it is not difficult to verify along the lines of [13, proof of Thm. 3.1.2, that for any value of the spectral parameter in the closure of the $n$th instability interval, $n \in \mathbb{Z}$, the Prüfer angle $\vartheta$ of any $\mathbb{R}^{2}$-valued solution $u$, defined by

$$
u=R\left(\begin{array}{c}
\cos \vartheta \\
-\sin \vartheta
\end{array}\right)
$$

with $R>0$, satisfies

$$
\vartheta(x)=\frac{n \pi x}{\alpha}+O(1) \quad(x \rightarrow \infty) .
$$

In particular, the asymptotic rate of growth of $\vartheta$, the so-called rotation number

$$
\lim _{x \rightarrow \infty} \frac{\vartheta(x)}{x}=\frac{n \pi}{\alpha},
$$

is constant in instability intervals.

It turns out that the rotation number is in fact well-defined as a continuous nondecreasing function of the spectral parameter on the whole real line, which can be expressed in terms of the discriminant in the stability intervals. For periodic SturmLiouville equations this was elegantly shown in [17], using a connection between the winding number of a complex-valued Floquet solution in the punctured plane and the Prüfer angle of its real part. Unfortunately, this argument does not carry over to the Dirac system, with Floquet solutions (in the case of stability) consisting of two complex-valued components with no immediate link between them. Nevertheless, the following statement holds. 
Theorem 2. Let $\vartheta$ be the Prüfer angle of an $\mathbb{R}^{2}$-valued solution of the Dirac system

$$
\left(-i \sigma_{2} \frac{d}{d x}+m \sigma_{3}+l \sigma_{1}+q\right) u=\lambda u
$$

with real-valued, $\alpha$-periodic coefficients $m, l, q \in L_{\mathrm{loc}}^{1}(\mathbb{R}), \lambda \in \mathbb{R}$ in a stability interval, and let $D$ be the discriminant.

Then there is $k(\lambda) \in \mathbb{R}$ such that $\vartheta(x)=\frac{k(\lambda) x}{\alpha}+O(1)(x \rightarrow \infty)$, and $D=$ $2 \cos k(\lambda)$.

$k(\lambda)$ is called the quasimomentum of this periodic equation; $k(\lambda) / \alpha$ is the rotation number. $k(\lambda) /(\alpha \pi)$ is called the integrated density of states in view of the following consequence of Theorem 2 , which is readily obtained using oscillation theory $([27]$, Thm. 14.7, 14.8).

Corollary 1. In the situation of Theorem 2 , let $\left(a_{n}\right)_{n \in \mathbb{N}},\left(b_{n}\right)_{n \in \mathbb{N}}$ be sequences of real numbers such that $a_{n}<b_{n}$ and $\lim _{n \rightarrow \infty} b_{n}-a_{n}=\infty$, and let $t_{n}$ be any self-adjoint realisation of

$$
-i \sigma_{2} \frac{d}{d x}+m \sigma_{3}+l \sigma_{1}+q
$$

on $\left[a_{n}, b_{n}\right]$ with separated boundary conditions. Then for $\lambda_{1}<\lambda_{2}$ the number $N_{n}$ of eigenvalues of $t_{n}$ in $\left[\lambda_{1}, \lambda_{2}\right]$ satisfies

$$
\lim _{n \rightarrow \infty} \frac{N_{n}}{b_{n}-a_{n}}=\frac{k\left(\lambda_{2}\right)-k\left(\lambda_{1}\right)}{\alpha \pi} .
$$

In the proof of Theorem 2 we use the following elementary observation on complex solutions of general Dirac systems with real-valued coefficients.

Lemma 1. Let $u: I \rightarrow \mathbb{C}^{2}$ be a non-trivial solution of

$$
\left(-i \sigma_{2} \frac{d}{d x}+m \sigma_{3}+l \sigma_{1}+q\right) u=\lambda u
$$

$\left(m, l, q \in L_{\mathrm{loc}}^{1}(I)\right.$ real-valued, $\left.\lambda \in \mathbb{R}\right)$. Then the following statements are equivalent:

a) There is $x \in I, \varphi \in \mathbb{C},|\varphi|=1$, such that $\varphi u(x) \in \mathbb{R}^{2}$.

b) There is $\varphi \in \mathbb{C},|\varphi|=1$, such that $\varphi u: I \rightarrow \mathbb{R}^{2}$.

c) $u, \bar{u}$ are linearly dependent.

Proof of Theorem 2. As $|D|<2$, the monodromy matrix has complex eigenvalues $\mu \neq \bar{\mu},|\mu|=1$. By Lemma 1, the corresponding Floquet solutions $u, \bar{u}$ are linearly independent as $\mu \notin \mathbb{R}$.

Again by Lemma 1 , the components $u_{1}, u_{2}$ have no zeros, and their arguments (locally absolutely continuous functions) are nowhere equal to mod $\pi$. By choosing an appropriate branch of the argument, we can assume $0<\left|\arg u_{1}-\arg u_{2}\right|<\pi$ throughout. More specifically, there are locally absolutely continuous functions $R_{1}, R_{2}>0, \varphi_{1}, \varphi_{2}$, and $\nu \in\{-1,1\}$ such that $u_{1}=R_{1} e^{i \varphi_{1}}, u_{2}=i \nu R_{2} e^{i \varphi_{2}}$, and $\left|\varphi_{1}-\varphi_{2}\right|<\pi / 2$. Interchanging $u$ and $\bar{u}$ if necessary, we can assume without loss of generality that $\nu=1$.

Consider the $\mathbb{R}^{2}$-valued solution $v:=\operatorname{Re} u$; its Prüfer angle satisfies

$$
\tan \vartheta=-\frac{v_{2}}{v_{1}}=\frac{R_{2} \sin \varphi_{2}}{R_{1} \cos \varphi_{1}}=\frac{R_{2}}{R_{1}} \cos \left(\varphi_{2}-\varphi_{1}\right)\left(\tan \varphi_{1}+\tan \left(\varphi_{2}-\varphi_{1}\right)\right) .
$$


Thus $\vartheta$ and $\varphi_{1}$ are connected by a modified Kepler transformation (cf. 25]); as a consequence, they take the values $(\mathbb{Z}+1 / 2) \pi$ at the same points, and their difference is globally bounded.

From $u_{1}(x+\alpha)=\mu u_{1}(x)$ we find that $R_{1}$ is $\alpha$-periodic, and that there is $k \in \mathbb{R}$ such that $\mu=e^{i k}$ and $\varphi_{1}(x+\alpha)=\varphi_{1}(x)+k(x \in \mathbb{R})$. Thus $\varphi_{1}(x)-\frac{k x}{\alpha}$ is $\alpha$-periodic and continuous, hence globally bounded, and it follows that

$$
\vartheta(x)=\frac{x k}{\alpha}+O(1) \quad(x \rightarrow \infty) .
$$

Furthermore, $D=\mu+\bar{\mu}=2 \cos k$.

\section{Proof of Theorem 1}

We shall use the following consequences of the spectral theorem (cf. [24, Lemma $6)$.

Lemma 2. Let $L$ be a self-adjoint operator with purely discrete spectrum of finite total multiplicity $N_{I}(L)$ in the real interval $I=\left[\lambda_{1}, \lambda_{2}\right]$.

a) (Decomposition Principle) If $L$ is a $k$-dimensional extension of a restriction of a self-adjoint operator $L^{\prime}$, then $N_{I}(L) \leq N_{I}\left(L^{\prime}\right)+k$.

b) (Bounded Perturbations) If $A$ is symmetric and bounded, then $N_{I}(L) \leq$ $N_{I^{\prime}}(L+A)$, where $I^{\prime}=\left[\lambda_{1}-\|A\|, \lambda_{2}+\|A\|\right]$.

The proof of Theorem 1 proceeds as follows. We introduce the rescaled independent variable $\varrho=r / c \in(0, \infty)$, and use the decomposition principle to split off the two regions near the singular endpoints 0 and $\infty$. The regular operator on the intermediate interval is then, by another application of the decomposition principle, split into operators on a large but finite number of subintervals on which the perturbation $\sigma_{1} l_{0}(\varrho)$ does not change very much. Lemma $2 \mathrm{~b}$ ) relates the number of eigenvalues of each subinterval operator to that of an operator with periodic coefficients on the same subinterval. By virtue of Corollary 1, the asymptotic number of eigenvalues for the latter can be expressed by means of the quasimomentum, considering that in terms of the original variable $r$, the subintervals grow beyond all bounds in the limit $c \rightarrow \infty$.

The operator on the unbounded interval near $\infty$, on which the perturbation is small, is easily dealt with in a similar manner. The endpoint 0 , however, presents more serious difficulties, which reflect the lack of monotonicity of spectral behaviour with respect to a matrix perturbation; it is at this point only that our rather strong hypotheses $(\mathrm{H})$ enter. We show that with a suitably chosen boundary condition, the operator on the interval near 0 has no eigenvalues at all in $\left[\lambda_{1}, \lambda_{2}\right]$ for sufficiently large $c$. The asymptotic formula then follows in the limit of infinite refinement of the subintervals.

The Dirac system

$$
\tau(c) u=\lambda u
$$

is in the limit point case at infinity ([27], Cor. to Theorem 6.8). We begin the proof of Theorem 1 by showing that for sufficiently large $c$, it is in the limit point case at 0 as well, thus proving the essential self-adjointness.

Let $\varrho$ $>0$ and $C>0$ be such that $l_{0}(\varrho)>0(\varrho \in(0, \hat{\varrho}))$ and

$$
\frac{\left|l_{0}^{\prime}(\varrho)\right|}{l_{0}(\varrho)^{2}} \leq C \quad(\varrho \in(0, \hat{\varrho})) \text {. }
$$


Then $\left(-\log l_{0}\right)^{\prime}(\varrho) \leq C l_{0}(\varrho)$, and hence

$$
l_{0}(\varrho) \leq l_{0}(\varrho) \exp \left(C \int_{\varrho}^{\varrho} l_{0}\right) \quad(\varrho \in(0, \hat{\varrho}))
$$

similarly

$$
l_{0}(\varrho)-l_{0}(\varrho)=-\int_{\varrho}^{\hat{\varrho}} l_{0}^{\prime} \leq C \int_{\varrho}^{\hat{\varrho}} l_{0}^{2},
$$

which implies $l_{0} \notin L^{2}(0, \cdot)$ in view of $\lim _{\varrho \rightarrow 0} l_{0}(\varrho)=\infty$.

As $m \sigma_{3}+q-\lambda$ is bounded, it is sufficient to establish that

$$
\left(-i \sigma_{2} \frac{d}{d r}+\sigma_{1} l_{0}(r / c)\right) u=0
$$

is in the limit point case at 0 . If $u$ is a non-trivial $\mathbb{R}^{2}$-valued solution of this equation, then $u_{1}^{\prime}(r)=-l_{0}(r / c) u_{1}(r)$, and setting $v(\varrho):=u_{1}(c \varrho)$, we find for $c \geq C$

$$
v(\varrho)=v(\hat{\varrho}) \exp \left(c \int_{\varrho}^{\varrho} l_{0}\right) \geq \frac{v(\varrho)}{l_{0}(\hat{\varrho})} l_{0}(\varrho)
$$

so $v \notin L^{2}(0, \cdot)$.

To prove the asymptotic formula, we first observe that the region near 0 does not contribute to the eigenvalue count. Indeed, let $c_{0}:=C+1$. Then

$$
l_{0}^{2}(\varrho)-\frac{1}{c_{0}}\left|l_{0}^{\prime}(\varrho)\right| \geq \frac{l_{0}^{2}(\varrho)}{c_{0}} \rightarrow \infty \quad(\varrho \rightarrow 0),
$$

and thus there is $\varrho_{0}>0$ such that

$$
l_{0}^{2}(\varrho)-\frac{1}{c_{0}}\left|l_{0}^{\prime}(\varrho)\right| \geq\left(\|q\|_{\infty}+\max \left\{\left|\lambda_{1}\right|,\left|\lambda_{2}\right|\right\}+1\right)^{2} \quad\left(\varrho \in\left(0, \varrho_{0}\right)\right) .
$$

Let $c \geq c_{0}$, and let $h_{0}(c)$ be the self-adjoint realisation of $\tau(c)$ on $\left(0, c \varrho_{0}\right)$ with the boundary condition

$$
u_{1}\left(c \varrho_{0}\right)+u_{2}\left(c \varrho_{0}\right)=0 .
$$

As $\tau(c)$ is in the limit point case at $0, h_{0}(c)$ is essentially self-adjoint on $D_{0}:=\{u \in$ $D\left(h_{0}(c)\right) \mid u \equiv 0$ near 0$\}$. Hence if $\lambda \in \sigma\left(h_{0}(c)\right)$ and $\varepsilon \in(0,1)$, there is $u \in D_{0} \backslash\{0\}$ such that $\left\|\left(h_{0}(c)-\lambda\right) u\right\|<\varepsilon\|u\|$, so

$$
\left\|-i \sigma_{2} u^{\prime}+\left(m \sigma_{3}+l_{0}(\cdot / c) \sigma_{1}\right) u\right\| \leq\left(\|q\|_{\infty}+|\lambda|+\varepsilon\right)\|u\| .
$$

But on the other hand, integration by parts yields

$$
\begin{aligned}
& \left\|-i \sigma_{2} u^{\prime}+\left(m \sigma_{3}+l_{0}(\cdot / c) \sigma_{1}\right) u\right\|^{2} \\
& \quad=\int_{0}^{c \varrho_{0}}\left(\left|u^{\prime}\right|^{2}+\left(m^{2}+l_{0}(\cdot / c)^{2}\right)|u|^{2}-m\left(\left(\sigma_{1} u\right)^{T} \bar{u}\right)^{\prime}+l_{0}(\cdot / c)\left(\left(\sigma_{3} u\right)^{T} \bar{u}\right)^{\prime}\right) \\
& \quad=m\left|u\left(c \varrho_{0}\right)\right|^{2}+\int_{0}^{c \varrho_{0}}\left(\left|u^{\prime}\right|^{2}+\left(m^{2}+l_{0}(\cdot / c)^{2}\right)|u|^{2}-\frac{1}{c} l_{0}^{\prime}(\cdot / c)\left(\left(\sigma_{3} u\right)^{T} \bar{u}\right)\right) \\
& \quad \geq \int_{0}^{c \varrho_{0}}\left(l_{0}(r / c)^{2}-\frac{1}{c}\left|l_{0}^{\prime}(r / c)\right|\right)|u(r)|^{2} d r \geq\left(\|q\|_{\infty}+\max \left\{\left|\lambda_{1}\right|,\left|\lambda_{2}\right|\right\}+1\right)^{2}\|u\|^{2} .
\end{aligned}
$$

Thus $|\lambda|>\max \left\{\left|\lambda_{1}\right|,\left|\lambda_{2}\right|\right\}$; in particular, $h(c)$ has no spectrum in $\left[\lambda_{1}, \lambda_{2}\right]$. 
A similar calculation shows that for all fixed $\varrho \in\left(0, \varrho_{0}\right)$, the self-adjoint periodic operator

$$
-i \sigma_{2} \frac{d}{d x}+m \sigma_{3}+q(x)+l_{0}(\varrho) \sigma_{1}
$$

has a spectral gap, and hence instability interval, containing $\left[\lambda_{1}, \lambda_{2}\right]$, and it follows that $k\left(\lambda_{1}, l_{0}(\varrho)\right)=k\left(\lambda_{2}, l_{0}(\varrho)\right)\left(\varrho \in\left(0, \varrho_{0}\right)\right)$.

We now turn to the remaining interval $\left(c \varrho_{0}, \infty\right)$. Choose $\delta_{\infty} \in(0,1)$ so small that $\delta_{\infty}<\left(\lambda_{2}-\lambda_{1}\right) / 2$, and $\left[\lambda_{1}-\delta_{\infty}, \lambda_{2}+\delta_{\infty}\right]$ is still compactly contained in the spectral gap of the periodic problem. Fix $P_{0}>\varrho_{0}$ such that $\left|l_{0}(\varrho)\right|<\delta_{\infty}\left(\varrho>P_{0}\right)$.

Let $\varepsilon>0$. There is $L>0$ such that $\left|l_{0}(\varrho)\right| \leq L\left(\varrho \in\left(\varrho_{0}, \infty\right)\right)$. As the quasimomentum $k(\lambda, l)$ is uniformly continuous on the compact set $K:=\left[\lambda_{1}-1, \lambda_{2}+1\right] \times$ $[-L, L]$, there is $\delta \in\left(0, \delta_{\infty}\right)$ such that

$$
(\mu, l),\left(\mu^{\prime}, l^{\prime}\right) \in K,\left|\mu-\mu^{\prime}\right|,\left|l-l^{\prime}\right|<\delta \Rightarrow\left|k(\mu, l)-k\left(\mu^{\prime}, l^{\prime}\right)\right|<\varepsilon .
$$

As $l_{0}$ is uniformly continuous on $\left[\varrho_{0}, P_{0}\right]$, there is $\gamma>0$ such that

$$
\left|l_{0}(x)-l_{0}(y)\right|<\delta \quad\left(x, y \in\left[\varrho_{0}, P_{0}\right],|x-y|<\gamma\right) .
$$

Now consider a partitioning of the interval $I:=\left(\varrho_{0}, P_{0}\right)$ into $n$ subintervals $I_{j}=\left(\varrho_{j-1}, \varrho_{j}\right)$ with $\left|I_{j}\right|<\gamma(j \in\{1, \ldots, n\})$.

Let $h_{j}(c), j \in\{1, \ldots, n\}$, and $h_{\infty}(c)$ be self-adjoint realisations of $\tau(c)$ on $c I_{j}$ and $\left(c P_{0}, \infty\right)$, respectively, with separated boundary conditions at the regular endpoints. Then $h_{0}(c) \oplus \bigoplus_{j=1}^{n} h_{j}(c) \oplus h_{\infty}(c)$ is a $2(n+1)$-dimensional extension of the $2(n+1)$-dimensional restriction of $h(c)$ with domain $\left\{u \in D(h(c)) \mid u\left(c \varrho_{j}\right)=0(j \in\right.$ $\{0, \ldots, n\})\}$; comparing these operators by means of the decomposition principle (Lemma 2 a)) we find

$$
\left|N_{0}(c)+\sum_{j=1}^{n} N_{j}(c)+N_{\infty}(c)-N(c)\right| \leq 2(n+1)
$$

for the total spectral multiplicities in $\left[\lambda_{1}, \lambda_{2}\right]$. As observed above, $N_{0}(c)=0$.

To estimate $N_{j}(c)$ for $j \in\{1, \ldots, n\}$, choose $\tilde{\varrho}_{j} \in I_{j}$, let $l_{j}:=l_{0}\left(\tilde{\varrho}_{j}\right)$, and define self-adjoint operators

$$
\tilde{h}_{j}(c)=-i \sigma \frac{d}{d r}+m \sigma_{3}+q(r)+l_{j} \sigma_{1}
$$

on $c I_{j}$ with $D\left(\tilde{h}_{j}(c)\right)=D\left(h_{j}(c)\right)$. Then Lemma 2 b) and $\left|l_{0}(\varrho)-l_{j}\right|<\delta\left(\varrho \in I_{j}\right)$ yield the bounds

$$
\tilde{N}_{j}\left(\lambda_{1}+\delta, \lambda_{2}-\delta ; c\right) \leq N_{j}(c) \leq \tilde{N}_{j}\left(\lambda_{1}-\delta, \lambda_{2}+\delta ; c\right),
$$

where $\tilde{N}_{j}\left(\mu_{1}, \mu_{2} ; c\right)$ is the number of eigenvalues of $\tilde{h}_{j}(c)$ in $\left[\mu_{1}, \mu_{2}\right]$.

As $\tilde{h}_{j}(c)$ has $\alpha$-periodic coefficients, Corollary 1 implies

$$
\lim _{c \rightarrow \infty} \frac{\tilde{N}_{j}\left(\mu_{1}, \mu_{2} ; c\right)}{c\left|I_{j}\right|}=\frac{k\left(\mu_{2}, l_{j}\right)-k\left(\mu_{1}, l_{j}\right)}{\alpha \pi} .
$$


Thus we find

$$
\begin{aligned}
& \frac{\left|I_{j}\right|}{\alpha \pi}\left(k\left(\lambda_{2}, l_{j}\right)-k\left(\lambda_{1}, l_{j}\right)-2 \varepsilon\right) \leq \frac{\left|I_{j}\right|}{\alpha \pi}\left(k\left(\lambda_{2}-\delta, l_{j}\right)-k\left(\lambda_{1}+\delta, l_{j}\right)\right) \\
& \quad=\lim _{c \rightarrow \infty} \frac{1}{c} \tilde{N}_{j}\left(\lambda_{1}+\delta, \lambda_{2}-\delta ; c\right) \leq \liminf _{c \rightarrow \infty} \frac{N_{j}(c)}{c} \\
& \quad \leq \limsup _{c \rightarrow \infty} \frac{N_{j}(c)}{c} \leq \lim _{c \rightarrow \infty} \frac{1}{c} \tilde{N}_{j}\left(\lambda_{1}-\delta, \lambda_{2}+\delta ; c\right) \\
& \quad=\frac{\left|I_{j}\right|}{\alpha \pi}\left(k\left(\lambda_{2}+\delta, l_{j}\right)-k\left(\lambda_{1}-\delta, l_{j}\right)\right) \leq \frac{\left|I_{j}\right|}{\alpha \pi}\left(k\left(\lambda_{2}, l_{j}\right)-k\left(\lambda_{1}, l_{j}\right)+2 \varepsilon\right) .
\end{aligned}
$$

In order to estimate $N_{\infty}(c)$, we observe that

$$
\tilde{N}_{\infty}\left(\lambda_{1}+\delta_{\infty}, \lambda_{2}-\delta_{\infty} ; c\right) \leq N_{\infty}(c) \leq \tilde{N}_{\infty}\left(\lambda_{1}-\delta_{\infty}, \lambda_{2}+\delta_{\infty} ; c\right),
$$

where $\tilde{N}_{j}\left(\mu_{1}, \mu_{2} ; c\right)$ is the number of eigenvalues in $\left[\mu_{1}, \mu_{2}\right]$ of the self-adjoint realisation $\tilde{h}_{\infty}(c)$ of $\tau$ on $\left(c P_{0}, \infty\right)$ with $D\left(\tilde{h}_{\infty}(c)\right)=D\left(h_{\infty}(c)\right)$. Since $\tilde{h}_{\infty}(c)$ is a direct summand of a 2-dimensional extension of a restriction of the unperturbed periodic operator on $\mathbb{R}$, we find, again by the decomposition principle, that $0 \leq$ $\tilde{N}_{\infty}\left(\mu_{1}, \mu_{2} ; c\right) \leq 2$ for any $\left[\mu_{1}, \mu_{2}\right]$ compactly contained in a spectral gap.

Summing up, we obtain

$$
\begin{gathered}
\frac{1}{\alpha \pi} \sum_{j=1}^{n}\left(k\left(\lambda_{2}, l_{j}\right)-k\left(\lambda_{1}, l_{j}\right)\right)\left|I_{j}\right|-\frac{2 \varepsilon}{\alpha \pi}|I| \leq \liminf _{c \rightarrow \infty} \frac{1}{c} \sum_{j=1}^{n} N_{j}(c) \\
=\liminf _{c \rightarrow \infty} \frac{N(c)}{c} \leq \limsup _{c \rightarrow \infty} \frac{N(c)}{c}=\limsup _{c \rightarrow \infty} \frac{1}{c} \sum_{j=1}^{n} N_{j}(c) \\
\leq \frac{1}{\alpha \pi} \sum_{j=1}^{n}\left(k\left(\lambda_{2}, l_{j}\right)-k\left(\lambda_{1}, l_{j}\right)\right)\left|I_{j}\right|+\frac{2 \varepsilon}{\alpha \pi}|I|,
\end{gathered}
$$

and refining the Riemann sums,

$$
\begin{aligned}
& \frac{1}{\alpha \pi} \int_{I}\left(k\left(\lambda_{2}, l_{0}(\varrho)\right)-k\left(\lambda_{1}, l_{0}(\varrho)\right)\right) d \varrho-\frac{2 \varepsilon}{\alpha \pi}|I| \leq \liminf _{c \rightarrow \infty} \frac{N(c)}{c} \\
& \quad \leq \limsup _{c \rightarrow \infty} \frac{N(c)}{c} \leq \frac{1}{\alpha \pi} \int_{I}\left(k\left(\lambda_{2}, l_{0}(\varrho)\right)-k\left(\lambda_{1}, l_{0}(\varrho)\right)\right) d \varrho+\frac{2 \varepsilon}{\alpha \pi}|I| .
\end{aligned}
$$

The assertion of Theorem 1 follows, as $\varepsilon>0$ is arbitrary, and $k\left(\lambda_{2}, l_{0}(\varrho)\right)=$ $k\left(\lambda_{1}, l_{0}(\varrho)\right)(\varrho \in(0, \infty) \backslash I)$.

This concludes the proof of Theorem 1 .

\section{REFERENCES}

1. Alama S., Deift P.A., Hempel R., Eigenvalue branches of the Schrödinger operator $H-\lambda W$ in a gap of $\sigma(H)$, Commun. Math. Phys. 121 (1989) 291-321. MR 90e:35046

2. Birman M.Sh., Discrete spectrum in the gaps of the continuous one in the large-couplingconstant limit, in: Order, disorder and chaos in quantum systems (Dubna 1989), Oper. Theory Adv. Appl. 46, Birkhäuser, Basel 1990, pp. 17-25. MR 92j:47091

3. Birman M.Sh., Discrete spectrum in the gaps of a continuous one for perturbations with large coupling limit, Adv. Sov. Math. 7 (1991) 57-73. MR 95h:47009

4. Birman M.Sh., On a discrete spectrum in gaps of a second order perturbed periodic operator, Funct. Anal. Appl. 25 (2) (1991) 158-161. MR 92m:47090

5. Birman M.Sh., The discrete spectrum in gaps of the perturbed periodic Schrödinger operator I. Regular perturbations, in: Boundary value problems, Schrödinger operators, deformation quantization. Math. Top., 8, Akademie Verlag, Berlin 1995, pp. 334-352. MR 97d:47055 
6. Birman M.Sh., The discrete spectrum of the periodic Schrödinger operator perturbed by a decreasing potential, St. Petersburg Math. J. 8 (1) (1997) 1-14. MR 97h:47047

7. Birman M.Sh., The discrete spectrum in gaps of the perturbed periodic Schrödinger operator II. Nonregular perturbations., St. Petersburg Math. J. 9 (6) (1998) 1073-1095. MR 99h:47054

8. Birman M.Sh., Laptev A., Discrete spectrum of the perturbed Dirac operator, Ark. Mat. 32 (1994) 13-32. MR 95h:35162

9. Birman M.Sh., Laptev A., The negative discrete spectrum of a two-dimensional Schrödinger operator, Comm. Pure Appl. Math. 49 (1996) 967-997. MR 97i:35131

10. Birman M.Sh., Laptev A., Solomyak M., The negative discrete spectrum of the operator $-\Delta^{l}-$ $\alpha V$ in $L_{2}\left(\mathbb{R}^{d}\right)$ for $d$ even and $2 l \geq d$, Ark. Mat. 35 (1997) 87-126. MR 98d:35167

11. Brown B.M., Eastham M.S.P., Hinz A.M., Schmidt K.M., Distribution of eigenvalues in gaps of the essential spectrum of Sturm-Liouville operators - a numerical approach, J. Comp. Anal. Appl. (to appear)

12. Cancelier C., Lévy-Bruhl P., Nourrigat J., Remarks on the spectrum of Dirac operators, Acta Appl. Math. 45 (1996) 349-364. MR 97m:35195

13. Eastham M.S.P., The spectral theory of periodic differential equations, Scottish Academic Press, Edinburgh 1973.

14. Hempel R. Herbst I., Hinz A.M., Kalf H, Intervals of dense point spectrum for spherically symmetric Schrödinger operators of the type $-\Delta+\cos |x|$, J. London Math. Soc. (2) 43 (1989) 295-304. MR 92f:35109

15. Klaus M., On the point spectrum of Dirac operators, Helv. Phys. Acta 53 (1980) 453-462. MR 83e: 81022

16. Laptev A., Asymptotics of the negative discrete spectrum of a class of Schrödinger operators with large coupling constant, Proc. Amer. Math. Soc. (2) 119 (1993) 481-488. MR 93k:35199

17. Moser J., An example of a Schrödinger equation with almost periodic potential and nowhere dense spectrum, Comment. Math. Helv. 56 (1981) 198-224. MR 82k:34029

18. Reed M, Simon B., Methods of modern mathematical physics IV: Analysis of operators, Academic Press, New York 1978. MR 58:12429c

19. Rofe-Beketov F.S., Spectral analysis of the Hill operator and of its perturbations, Functional Analysis 9 (1977) 144-155 (Russian). MR 58:12520

20. Rofe-Beketov F.S., A generalisation of the Prüfer transformation and the discrete spectrum in gaps of the continuous spectrum, in: Spectral theory of operators., Elm, Baku 1979, pp. 146-153 (Russian). MR 81i:34021

21. Rofe-Beketov F.S., Spectrum perturbations, the Kneser-type constants and the effective masses of zones-type potentials, Sofia 1984, pp. 757-766.

22. Rofe-Beketov F.S., Kneser constants and effective masses for band potentials, Sov. Phys. Dokl. 29 (5) (1984) 391-393. MR 86c:34054

23. Schmidt K.M., On the essential spectrum of Dirac operators with spherically symmetric potentials, Math. Ann. 297 (1993) 117-131. MR 94j:35114

24. Schmidt K.M., Dense point spectrum and absolutely continuous spectrum in spherically symmetric Dirac operators, Forum Math. 7 (1995) 459-475. MR 96f:47097

25. Schmidt K.M., Critical coupling constants and eigenvalue asymptotics of perturbed periodic Sturm-Liouville operators, Commun. Math. Phys. 211 (2000) 465-485. MR 2001i:34147

26. Sobolev A.V., Weyl asymptotics for the discrete spectrum of the perturbed Hill operator, Adv. Sov. Math. 7 (1991) 159-178. MR 95i:34158

27. Weidmann J., Spectral theory of ordinary differential operators, Lect. Notes in Math. 1258, Springer, Berlin 1987. MR 89b:47070

School of Mathematics, Cardiff University, 23 Senghennydd Rd., Cardiff CF2 4 4YH, UNITED KINGDOM

E-mail address: SchmidtKM@Cardiff.ac.uk 